\title{
DESEMPENHO DE PONTAS DE PULVERIZAÇÃO EM Brachiaria brizantha cv. MG-4 PARA CONTROLE DE NINFAS DE CIGARRINHAS DAS PASTAGENS
}

\section{CLEBER D. DE G. MACIEL ${ }^{1}$, EDIVALDO D. VELINI ${ }^{2}$, RODRIGO DOS S. BERNARDO ${ }^{3}$}

RESUMO: O trabalho teve como objetivo estudar o desempenho de pontas de pulverização na deposição da calda inseticida para o controle de ninfas de cigarrinhas das pastagens em Brachiaria brizantha cv. MG-4. Doze tratamentos foram estudados em esquema fatorial 6x2, constituídos pelo contraste de seis pontas de pulverização e pressões de 196 e $392 \mathrm{kPa}$ : TF-VP2 (336 L ha ${ }^{-1}$ e $\left.467 \mathrm{~L} \mathrm{ha}^{-1}\right)$; AI11002-VS (184 L ha ${ }^{-1}$ e $\left.200 \mathrm{~L} \mathrm{ha}^{-1}\right)$; XR11002-VS (200 L ha ${ }^{-1}$ e $\left.280 \mathrm{~L} \mathrm{ha}^{-1}\right)$; TT11002-VP (200 L ha e $\left.280 \mathrm{~L} \mathrm{ha}^{-1}\right)$; TJ60-11002VS (208 L ha ${ }^{-1}$ e $280 \mathrm{~L} \mathrm{ha}^{-1}$ ) e TX-VK4 (72 $\mathrm{L} \mathrm{ha}^{-1}$ e $\left.97 \mathrm{~L} \mathrm{ha}^{-1}\right)$. Para monitorar a deposição das caldas de pulverização, utilizaram-se os traçadores Azul Brilhante FD\&C-1 (0,3\% p/v) e Amarelo de Tartrasina FD\&C-5 (0,6\% p/v). Alvos artificiais, constituídos de lâminas de vidro, foram posicionados na base das plantas, próximos à superfície do solo, e os depósitos por unidade de área das soluções pulverizadas foram quantificados por espectrofotometria. As pontas TF-VP2, XR11002-VS e AI11002-VS, nas pressões de 196 e $392 \mathrm{kPa}$, proporcionam as maiores deposições da calda de pulverização na região das espumas das cigarrinhas das pastagens, apesar de apresentarem menor uniformidade na distribuição dos depósitos em relação a TX-VK4, XR110.02-VS e TJ110.02-VS. O aumento da pressão de 196 para $392 \mathrm{kPa}$ promoveu aumento na deposição da calda de pulverização sobre a Brachiaria brizantha e na região onde se encontram as espumas das cigarrinhas para todos os tipos de pontas estudadas.

PALAVRAS-CHAVE: pontas de pulverização, volume de calda, deposição, forrageira.

\section{SPRAY NOZZLES PERFORMANCE IN Brachiaria brizantha cv. MG-4 FOR PASTURES SPITTLEBUGS NYMPHS CONTROL}

\begin{abstract}
The work aimed to study spray nozzles performance in pesticide sprayer deposition for controlling pastures spittlebugs nymphs in Brachiaria brizantha cv. MG-4 pasture. Twelve treatments were studied in factorial scheme $6 \times 2$, constituted by the contrast of six spray nozzles and 196 and $392 \mathrm{kPa}$ work pressures: TF-VP2 (336 L ha ${ }^{-1}$ and $467 \mathrm{~L} \mathrm{ha}^{-1}$ ); AI11002-VS (184 L ha ${ }^{-1}$ and $\left.200 \mathrm{~L} \mathrm{ha}^{-1}\right)$; XR11002-VS $\left(200 \mathrm{~L} \mathrm{ha}^{-1}\right.$ and $\left.280 \mathrm{~L} \mathrm{ha}^{-1}\right)$; TT11002-VP (200 L ha ${ }^{-1}$ and $280 \mathrm{~L} \mathrm{ha}^{1}$ ); TJ60-11002VS (208 L ha ${ }^{-1}$ and $\left.280 \mathrm{~L} \mathrm{ha}^{-1}\right)$ and TX-VK4 (72 L ha ${ }^{-1}$ and $\left.97 \mathrm{~L} \mathrm{ha}^{-1}\right)$. For monitoring sprayer deposition it was used Bright Blue FD\&C-1 $(0.3 \%$ p/v) and Tartrasine Yellow FD\&C-5 $(0.6 \% \mathrm{p} / \mathrm{v})$ as tracers. Artificial targets, constituted by thin glass plates were positioned on plants tillers base, near to soil surface and deposition per unit area of sprayed solutions were quantified by spectrophotometry. TF-VP2; XR11002-VS and AI11002-VS spray nozzles, in 196 and $392 \mathrm{kPa}$ work pressures provided the largest sprayer deposition in the foam regions of pastures spittlebugs nymph, in spite of showing inferior uniformities of sprayer deposition in relation to TX-VK4, XR110.02-VS and TJ110.02-VS.The increase of work pressure from 196 to $392 \mathrm{kPa}$ promoted the increase of sprayer deposition over Brachiaria brizantha and over the region where pastures spittlebugs foams were located for all types of spray nozzles studied.
\end{abstract}

KEYWORDS: spray nozzles, application tax, deposition, forage.

\footnotetext{
${ }^{1}$ Eng ${ }^{\mathrm{o}}$ Agrônomo, Prof. Dr., Departamento de Ciências Biológicas e Fitossanitárias, Escola Superior de Agronomia de Paraguaçu Paulista, ESAPP, Paraguaçu Paulista - SP, maciel@ fca.unesp.br

${ }^{2}$ Eng ${ }^{\circ}$ Agrônomo, Prof. Dr., Departamento de Produção Vegetal - Agricultura, Faculdade de Ciências Agronômicas, UNESP, Botucatu - SP.

${ }^{3}$ Eng $^{\mathrm{O}}$ Agrônomo, Empresa Zoofort Agrícola \& Animal, Primavera do Leste - MT.

Recebido pelo Conselho Editorial em: 3-3-2005
}

Aprovado pelo Conselho Editorial em: 19-9-2006 


\section{INTRODUÇÃO}

As gramíneas têm sido o principal alimento da pecuária brasileira desde a introdução no Brasil, pois a maior parte do rebanho nacional é mantida exclusivamente a pasto, que é um alimento barato, disponível e de fácil manejo, caracterizado por não haver necessidade de colheita, armazenamento e distribuição.

Segundo ZIMMER \& CORREA (1993), no Brasil tropical-subtropical, anualmente, são semeados cerca de 5,5 milhões de hectares de pastagens perenes, incluindo áreas de formação, recuperação e renovação. As braquiárias (Brachiaria decumbens, B. brizantha e B. humidicola) correspondem acerca de $80 \%$ do mercado de sementes forrageiras, onde somente a demanda por $B$. brizantha perfaz mais de $50 \%$. Verifica-se, nas estimativas, que a área total de pastagens cultivadas correspondem acerca de 110 milhões de hectares, divididos pelas regiões Norte, Nordeste, Sul, Sudeste e Centro-Oeste, respectivamente, com 25; 14; 7; 20 e 44 milhões de hectares (SERRÃO et al., 1993; ZIMMER \& EUCLIDES FILHO, 1997; NABINGER et al., 1999).

Dentre os fatores limitantes para o desenvolvimento das pastagens brasileiras, destacam-se a ineficiência na ciclagem de nutrientes, as cigarrinhas das pastagens e a infestação por invasoras (SERRÃO et al., 1978; DIAS FILHO, 1998). Os danos econômicos causados pelas cigarrinhas dos gêneros Deois flavopicta, Zulia entreriana e Mahanarva fimbriolata são expressivos em pastagens cultivadas e em lavouras canavieiras, nas diferentes regiões do Brasil. ALVES (1984) e Silveira NETO (1994) relataram que as cigarrinhas podem provocar prejuízos, entre 10 e 90\%, em pastagens estabelecidas, dependendo das espécies forrageiras, condições climáticas e manejo da pastagem. Calcula-se que 25 cigarrinhas adultas por metro quadrado, em 10 dias, reduzam, em média, 30\% a produção forrageira do pasto atacado e causam prejuízos da ordem de 15\% na produção de massa verde (SILVEIRA NETO, 1994).

Segundo GALLO et al. (2002), as cigarrinhas são insetos sugadores de seiva, cujos adultos vivem na parte aérea das plantas, e suas ninfas ficam protegidas por espuma característica na base das plantas, próximo das raízes. Por serem altamente fitotoxicogênicas, podem causar reduções de até $60 \%$ na capacidade de suporte das pastagens (MELO \& AZEVEDO, 1998).

Propostas para o manejo integrado das cigarrinhas das pastagens incluem a diversificação, o uso de espécies resistentes, o manejo adequado da carga animal, o controle biológico, a queimada controlada em áreas com histórico de altas infestações e o controle químico, quando forem encontradas entre 20 a 25 ninfas por metro quadrado (ALVES, 1984; PEREIRA, 1990; VALÉRIO \& KOLLER, 1995; ALMEIDA \& NASCIMENTO JÚNIOR, 2004). Nesse contexto, medidas preventivas que integram controle químico e biológico vêm sendo adotadas para a manutenção dos níveis populacionais abaixo do nível de dano econômico. Entretanto, ainda são restritas as informações sobre as melhores condições operacionais das aplicações de inseticidas em pastagens, principalmente no que se refere à qualidade da deposição da calda de pulverização nas espumas, onde se encontram as ninfas das cigarrinhas e, conseqüentemente, na própria pastagem.

Para HISLOP et al. (1987), os maiores objetivos em pesquisas com aplicação de produtos fitossanitários são a definição da deposição em alvos biológicos e a identificação de métodos precisos de aplicação, que são desenvolvidos para permitir a redução no volume de pulverização e/ou dose do ingrediente ativo, sem perda da eficácia biológica. Nesse contexto, a tecnologia de aplicação inadequada é um dos fatores limitantes à eficácia dos produtos fitossanitários, tornando necessário o constante aprimoramento das técnicas de aplicação e de equipamentos. Segundo RAMOS \& PIO (2003), as pontas hidráulicas de pulverização têm como funções muito importantes a determinação da vazão, a distribuição e o tamanho de gotas, que são dependentes da pressão de trabalho, a qual é a fonte de energia para a formação das gotas.

Dessa forma, o trabalho teve como objetivo avaliar a eficácia de deposição da calda de pulverização proporcionada por modelos de pontas de pulverização, visando ao controle de ninfas de cigarrinhas das pastagens em Brachiaria brizantha cv. MG-4. 


\section{MATERIAL E MÉTODOS}

O trabalho foi realizado no período de 2003-2004, em pastagem de Brachiaria brizantha cv. MG-4, semeada a lanço e localizada na Fazenda San Martin, localizada no Município de Paraguaçu Paulista - SP. O delineamento experimental utilizado foi o inteiramente casualizado, com 12 tratamentos dispostos no esquema fatorial $6 \times 2$, representado pelo contraste de seis modelos de pontas de pulverização, trabalhando em duas condições de pressão (196 e $392 \mathrm{kPa})$. Os tratamentos testados foram: TF-VP2 (336 $\mathrm{L} \mathrm{ha}^{-1}$ e $\left.467 \mathrm{~L} \mathrm{ha}^{-1}\right)$, AI11002-VS (184 $\mathrm{L} \mathrm{ha}^{-1}$ e $\left.280 \mathrm{~L} \mathrm{ha}^{-1}\right)$, XR11002-VS (200 L ha' ${ }^{-1}$ e $\left.280 \mathrm{~L} \mathrm{ha}^{-1}\right)$, TT11002-VP (200 L ha ${ }^{-1}$ e $\left.280 \mathrm{~L} \mathrm{ha}^{-1}\right)$, TJ60-11002VS (208 L ha ${ }^{-1}$ e $\left.280 \mathrm{~L} \mathrm{ha}^{-1}\right)$ e TX-VK4 $\left(72 \mathrm{~L} \mathrm{ha}^{-1}\right.$ e $\left.97 \mathrm{~L} \mathrm{ha}^{-1}\right)$.

Para o monitoramento da deposição da calda de pulverização, assim como do aproveitamento dos mesmos alvos para as duas pressões estudadas com as pontas de pulverização, foram utilizados como traçadores os corantes alimentícios Azul Brilhante FD\&C-1 (0,3\% p/v) e Amarelo de Tartrasina FD\&-5 (0,6\% p/v), em pulverizações individualizadas e sobre os mesmos alvos, segundo metodologia descrita por PALLADINI (2000) e SOUZA (2002). As repetições foram constituídas por alvos artificiais, representadas por 15 lâminas de vidro por tratamento, com dimensões de 2,6 x 7,6 cm $\left(19,76 \mathrm{~cm}^{2}\right)$, posicionados aleatoriamente na base das touceiras das plantas, próximo à superfície do solo, simulando a localização das espumas produzidas pelas ninfas das cigarrinhas. Além disso, também foram consideradas como repetições de alvos naturais de 25 perfilhos individualizados das plantas da pastagem por tratamento. Após as pulverizações, os perfilhos foram coletados e divididos em porção superior e inferior da parte aérea das plantas.

A pulverização das parcelas foi realizada utilizando-se de pulverizador costal à pressão constante, proporcionada por $\mathrm{CO}_{2}$ comprimido, trabalhando em velocidade de $3,6 \mathrm{~km} \mathrm{~h}^{-1}$, com barra equipada com duas pontas espaçadas de $50 \mathrm{~cm}$ e a $50 \mathrm{~cm}$ de altura das plantas. No momento das aplicações, as plantas da pastagem encontravam-se perfilhadas, com aproximadamente $40 \mathrm{~cm}$ de altura, e os parâmetros meteorológicos médios registrados foram temperatura, umidade relativa do ar e velocidade do vento em $26^{\circ} \mathrm{C}, 69 \%$ e $2,1 \mathrm{~km} \mathrm{~h}^{-1}$, respectivamente.

O procedimento de recuperação do traçador foi realizado por meio da lavagem dos mesmos com volumes de 20 e $50 \mathrm{~mL}$ de água destilada, respectivamente, os quais foram agitados manualmente e de forma constante por 20 segundos dentro de sacos plásticos. A determinação das quantidades dos traçantes depositadas, em cada amostra, foi realizada em espectrofotômetro de duplo feixe, modelo Cintra 40, operando com $10 \mathrm{~mm}$ de caminho óptico, cujos resultados, em absorbância nos comprimentos de onda a $630 \mathrm{~nm}$ (FD\&C-1) e $427 \mathrm{~nm}$ (FD\&C-5), foram transformados em $\mathrm{mg} \mathrm{L}^{-1}$, de acordo com coeficiente angular da curva-padrão, similarmente à metodologia utilizada por PALLADINI (2000), SILVA (2000), TOMAZELA (2000) e SOUZA (2002).

As concentrações dos depósitos nas lâminas de vidro foram transformadas em volume por área $\left(\mu \mathrm{L} \mathrm{cm}{ }^{-2}\right)$ e ajustados ao modelo de Gompertz para representar as freqüências acumuladas da deposição das caldas pulverizadas em percentagem, conforme VELINI (1995). Para os alvos naturais, os depósitos também foram transformados em volume por área $\left(\mu \mathrm{L} \mathrm{cm}^{-2}\right)$ e submetidos à análise de variância, e suas médias comparadas pelo teste de Tukey, a 5\% de probabilidade.

Modelo de Gompertz: $F=e^{\left[a-e^{(-b-c x)}\right]}$ em que,

F - frequiência acumulada, percentagem;

a - valor atribuído em 4,605170, de modo que ea = 100;

b - valor estimado pelo modelo;

c - valor estimado pelo modelo, e

$\mathrm{x}$ - depósitos, em $\mu \mathrm{L} \mathrm{cm}^{-2}$. 


\section{RESULTADOS E DISCUSSÃO}

As curvas da freqüência acumulada $(\%)$ referentes aos depósitos da calda de pulverização por unidade de área $\left(\mu \mathrm{L} \mathrm{cm}{ }^{-2}\right)$ das lâminas de vidro, as quais simularam a localização das espumas das cigarrinhas, ajustaram-se ao modelo de Gompertz (Figura 1) e apresentaram elevados coeficientes de determinação $\left(\mathrm{R}^{2}\right.$ entre 0,9564 e 0,9909 , Tabela 1$)$, independentemente da ponta e/ou pressões de trabalho utilizada.
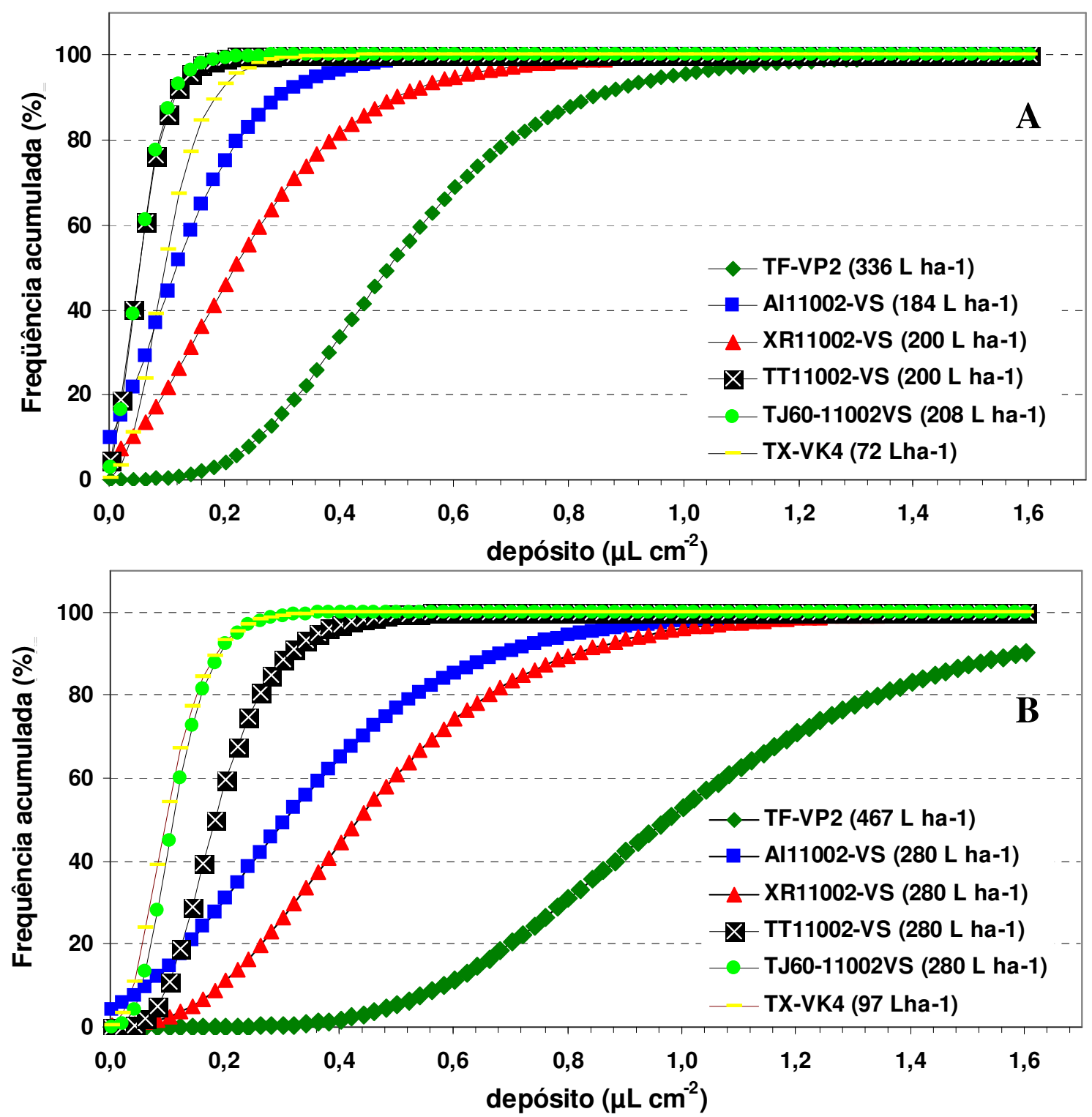

FIGURA 1. Freqüências acumuladas (\%) da deposição da calda de pulverização nas lâminas de vidro posicionadas na base das plantas de pastagem, em função dos tipos de pontas e pressões de pulverização de $196 \mathrm{kPa}$ (A) e 392 kPA (B), utilizando-se do modelo de Gompertz.

A distribuição da deposição da calda de pulverização nas lâminas de vidro apresentou maior uniformidade nas aplicações com pressão de $196 \mathrm{kPa}$, com exceção da ponta TX-VK4, a qual apresentou maior regularidade na distribuição da calda de pulverização sobre os alvos, utilizandose da pressão de $392 \mathrm{kPa}$. Isso é explicado pela diferença na inclinação das curvas representadas pelas Figuras 1A e 1B, comparando-se o comportamento de pontas iguais submetidas às diferentes pressões ou, ainda, por meio do parâmetro "c" do modelo de Gompertz (Tabela 1), em que os maiores valores indicam menores inclinações das curvas e maior uniformidade de deposição da calda de pulverização. Na Figura 1 e na Tabela 1, também se pode constatar que as pontas TJ6011002-VS, TT11002-VS e TX-VK4, apesar de terem proporcionado as maiores uniformidades de deposição (menores inclinações nas curvas e/ou maiores valores de "c"), foram as que 
proporcionaram as menores quantidades médias depositadas quando comparadas às pontas TFVP2, XR11002-VS e AI11002-VS, independentemente da pressão de trabalho

TABELA 1. Resultados das análises de regressão entre as frequiências acumuladas (\%) e os volumes de depósito, utilizando o modelo de Gompertz.

\begin{tabular}{|c|c|c|c|c|c|c|c|}
\hline \multicolumn{8}{|c|}{$\begin{array}{c}\text { Freqüência Acumulada (\%) - Depósito Utilizando Pressão de } 196 \text { kPa } \\
\text { Figura } 1 \text { (A) }\end{array}$} \\
\hline $\begin{array}{c}\text { Pontas de } \\
\text { Pulverização }\end{array}$ & & $\begin{array}{c}\text { TF-VP2 } \\
\left(336 \mathrm{~L} \mathrm{ha}^{-1}\right)\end{array}$ & $\begin{array}{l}\text { AI11002VS } \\
\left(184 \mathrm{~L} \mathrm{ha}^{-1}\right)\end{array}$ & $\begin{array}{l}\text { XR11002VS } \\
\left(200 \mathrm{~L} \mathrm{ha}^{-1}\right)\end{array}$ & $\begin{array}{l}\text { TT11002VS } \\
\left(200 \mathrm{~L} \mathrm{ha}^{-1}\right)\end{array}$ & $\begin{array}{l}\text { TJ60-11002VS } \\
\left(208 \mathrm{~L} \mathrm{ha}^{-1}\right)\end{array}$ & $\begin{array}{l}\text { TX-VK4 } \\
\left(72 \mathrm{~L} \mathrm{ha}^{-1}\right)\end{array}$ \\
\hline Estimativa dos & $\mathrm{A}$ & 4,6052 & 4,6052 & 4,6052 & 4,6052 & 4,6052 & 4,6052 \\
\hline Parâmetros & B & $-2,2311$ & $-0,8676$ & $-1,1186$ & $-1,1924$ & $-1,3302$ & $-1,1380$ \\
\hline & $\mathrm{C}$ & 5,3328 & 10,5044 & 6,7702 & 30,2484 & 32,6627 & 17,9044 \\
\hline Média $\left(\mu \mathrm{Lcm}^{-2}\right)$ & & 0,5283 & 0,1557 & 0,2509 & 0,0637 & 0,0642 & 0,1037 \\
\hline $\mathrm{F}$ & & $27343,13 * *$ & $27334,98 * *$ & $27416,58 * *$ & $27432,12 * *$ & $27512,11 * *$ & $27486,69 * *$ \\
\hline $\mathrm{R}^{2}$ & & 0,9718 & 0,9708 & 0,9816 & 0,9837 & 0,9877 & 0,9909 \\
\hline \multicolumn{8}{|c|}{$\begin{array}{c}\text { Freqüiência Acumulada (\%) - Depósito Utilizando Pressão de } 392 \text { kPa } \\
\text { Figura 1 (B) }\end{array}$} \\
\hline Pontas de & & TF-VP2 & AI11002VS & XR11002VS & TT11002VS & TJ60-11002VS & TX-VK4 \\
\hline Pulverização & & $\left(467 \mathrm{~L} \mathrm{ha}^{-1}\right)$ & $\left(280 \mathrm{~L} \mathrm{ha}^{-1}\right)$ & $\left(280 \mathrm{~L} \mathrm{ha}^{-1}\right)$ & $\left(280 \mathrm{~L} \mathrm{ha}^{-1}\right)$ & $\left(280 \mathrm{~L} \mathrm{ha}^{-1}\right)$ & $\left(97 \mathrm{~L} \mathrm{ha}^{-1}\right)$ \\
\hline Estimativa dos & A & 4,6052 & 4,6052 & 4,6052 & 4,6052 & 4,6052 & 4,6052 \\
\hline \multirow[t]{2}{*}{ Parâmetros } & B & $-2,6142$ & $-1,1606$ & $-1,7941$ & $-2,2812$ & $-2,1235$ & $-1,7036$ \\
\hline & $\mathrm{C}$ & 3,0606 & 4,9801 & 4,9778 & 14,4977 & 22,8663 & 21,4416 \\
\hline Média $\left(\mu \mathrm{Lcm}^{-2}\right)$ & & 1,0952 & 0,4205 & 0,4489 & 0,2421 & 0,1191 & 0,1225 \\
\hline $\mathrm{F}$ & & $27253,30 * *$ & $27371,20 * *$ & $27347,13 * *$ & $27417,34 * *$ & $27474,92 * *$ & $27226,44 * *$ \\
\hline $\mathrm{R}^{2}$ & & 0,9600 & 0,9756 & 0,9724 & 0,9817 & 0,9893 & 0,9564 \\
\hline
\end{tabular}

** = valor significativo pelo teste $\mathrm{F}$ a $\mathrm{P}<0,01$.

Entretanto, discrepância entre as maiores e as menores quantidades de calda de pulverização depositada por unidade de área das lâminas de vidro $\left(\mu \mathrm{L} \mathrm{cm}^{-2}\right)$, em função das diferentes pontas e pressões de trabalho, apresentou expressiva alteração. As diferenças entre os maiores e os menores depósitos para a pressão de 196, em relação a $392 \mathrm{kPa}$, para a mesma ponta, foram de 8,73 e 4,04; 69,43 e 21,30; 9,93 e 6,93; 27,10 e 18,07; 16,11 e 6,50; 43,34 e 7,78 vezes para os tipos TF-VP2, AI11002-VS, XR11002-VS, TT11002-VP, TJ60-11002VS e TX-VK4, respectivamente. Assim, o aumento da pressão de pulverização de 196 para $392 \mathrm{kPa}$ proporcionou redução acentuada da relação entre os maiores e menores depósitos de calda de pulverização em todos os tipos de pontas de pulverização. Nesse contexto, considerando que cada $1,0 \mu \mathrm{L} \mathrm{cm}^{-2}$ de depósito nas lâminas de vidro corresponde a $100 \mathrm{~L} \mathrm{ha}^{-1}$ de calda, o aumento da pressão de 196 para $392 \mathrm{kPa}$ pode favorecer o aumento da homogeneidade da deposição da calda de pulverização, devido à redução das diferenças entre as maiores e as menores quantidades de calda de pulverização depositadas.

Poder-se-ia exemplificar que, se a dose de inseticida mínima necessária para matar as ninfas de cigarrinhas estivesse contida em $0,2 \mu \mathrm{L} \mathrm{cm}^{-2}$ das espumas, haveria apenas 4,2\%; 99,3\%; 99,5\%; $46,0 \% ; 75,3$ e $92,0 \%$ (Figura 1A) e $0,1 \% ; 59,5 \% ; 92,2 \% ; 11,1 \% ; 31,2 \%$ e $93,1 \%$ (Figura 1B) de falhas de controle para as pontas TF-VP2, TT11002-VP, TJ60-11002VS, XR11002-VS, AI11002VS e TX-VK4, respectivamente, utilizando-se das pressões de pulverização de 196 e $392 \mathrm{kPa}$. Nesse exemplo, observa-se que o aumento da pressão de 196 para $392 \mathrm{kPa}$ promoveria considerável aumento na deposição da pulverização de inseticidas sobre as espumas localizadas na base das plantas de $B$. brizantha cv. MG-4, na ordem de 44,1\%;39,8\%; 34,9\%; 7,3\% e 4,1\%, utilizando-se das pontas AI11002-VS, TT11002-VP, XR11002-VS, TJ60-11002VS e TF-VP2, respectivamente.

Conforme informações do fabricante SPRAYING SYSTEMS Co. (1998 e 2000), o aumento da pressão de pulverização das pontas AI11002-VS, TT11002-VP, XR11002-VS e TJ60-11002VS promove a redução do tamanho das gotas durante a pulverização, sendo essas, respectivamente, classificadas pela escala da British Crop Protection Council (BCPC) como muito grossa, média, fina e muito fina. Dessa forma, considerando os aspectos qualitativos e de segurança nas aplicações 
dos produtos fitossanitários, observa-se que, para as pontas TJ60-11002VS, TT11002-VP e TX-V4, o aumento da pressão de pulverização e a conseqüente redução do tamanho das gotas (DMV), apesar de ter favorecido a penetração da calda de pulverização no dossel da pastagem até o local das espumas, podem promover maior perda por deriva.

Para o modelo TX-VK4, o aumento da pressão de trabalho dentro dos limites estudados, apesar de terem sido inferiores à recomendação do fabricante, considerada como acima de $392 \mathrm{kPa}$ (SPRAYING SYSTEMS Co., 1998 e 2000; RAMOS \& PIO, 2003), favoreceu apenas em 18,13\% o incremento médio da deposição sobre as lâminas de vidro (Tabela 1). Entretanto, as pontas XR11002-VS e TJ60-11002VS também proporcionariam perdas significativas por deriva, em função do aumento da quantidade produzida de gotas muito finas.

Na Tabela 2, observa-se que a deposição da calda de pulverização nas porções superior e inferior da parte aérea da pastagem também foi ampliada com o aumento da pressão de pulverização, com exceção do modelo TX-VK4. Essa ponta de pulverização proporcionou aumento da deposição da calda de pulverização na porção inferior dos perfilhos da pastagem. De forma geral, as interações entre os fatores pontas e pressão, apesar de terem sido significativas, ainda indicam dificuldade de penetração da calda de pulverização até as espumas das cigarrinhas, condição essa caracterizada no trabalho por meio da redução expressiva da deposição da calda de pulverização na porção inferior dos perfilhos da pastagem. Ressalta-se a significativa superioridade de deposição das porções superior e principalmente inferior dos perfilhos da pastagem para a ponta TF-VP2, nas pressões de 192 e $396 \mathrm{kPa}$, apesar de maior volume de calda ser aspecto negativo para a dinâmica operacional da pulverização.

TABELA 2. Deposição $\left(\mu \mathrm{L} \mathrm{cm}{ }^{-2}\right)$ média da calda de pulverização nas porções superior e inferior dos perfilhos da pastagem de $B$. brizantha $\mathrm{cv}$. MG-4.

\begin{tabular}{|c|c|c|c|c|}
\hline \multirow{3}{*}{ Tratamentos } & \multicolumn{4}{|c|}{ Deposição Média $\left(\mu \mathrm{L} \mathrm{cm}^{-2}\right)$} \\
\hline & \multicolumn{2}{|c|}{ Porção Superior } & \multicolumn{2}{|c|}{ Porção Inferior } \\
\hline & $196 \mathrm{kPa}$ & $392 \mathrm{kPa}$ & $196 \mathrm{kPa}$ & $392 \mathrm{kPa}$ \\
\hline TF-VP2 & $1,24 \mathrm{aB}$ & $3,57 \mathrm{aA}$ & $0,33 \mathrm{aB}$ & $0,94 \mathrm{aA}$ \\
\hline AI11002VS & $0,77 \mathrm{bB}$ & $2,17 \mathrm{cA}$ & $0,13 \mathrm{bB}$ & $0,39 \mathrm{bA}$ \\
\hline XR11002VS & $0,97 \mathrm{bB}$ & $2,51 \mathrm{bA}$ & $0,11 \mathrm{bB}$ & $0,32 \mathrm{bA}$ \\
\hline TT11002VS & $0,89 \mathrm{bB}$ & $2,34 \mathrm{bcA}$ & $0,11 \mathrm{bB}$ & $0,36 \mathrm{bA}$ \\
\hline TJ60-11002VS & $0,94 \mathrm{bB}$ & $2,52 \mathrm{bA}$ & $0,14 \mathrm{bB}$ & $0,40 \mathrm{bA}$ \\
\hline TX-VK4 & $0,46 \mathrm{cB}$ & $0,86 \mathrm{dA}$ & $0,10 \mathrm{bA}$ & $0,13 \mathrm{cA}$ \\
\hline Ponta (P) & \multicolumn{2}{|c|}{$248,42 * *$} & \multicolumn{2}{|c|}{$170,01 * *$} \\
\hline Pressão (OS) & \multicolumn{2}{|c|}{$2454,32 * *$} & \multicolumn{2}{|c|}{$567,69 * *$} \\
\hline $\mathrm{P} \times \mathrm{OS}$ & \multicolumn{2}{|c|}{$74,20 * *$} & \multicolumn{2}{|c|}{$46,02 * *$} \\
\hline C.V.\% & \multicolumn{2}{|c|}{15,79} & \multicolumn{2}{|c|}{33,95} \\
\hline
\end{tabular}

Médias seguidas de mesma letra maiúscula na linha e minúscula na coluna, para as porções superior e inferior das plantas, não diferem entre si, pelo teste Tukey, a 5\% de probabilidade. $* *=\mathrm{P}<0,01$.

Verifica-se, nestes resultados, comportamento distinto das pontas de pulverização em relação à formação das gotas e ao volume da aplicação, os quais influenciam na capacidade de penetração da pulverização entre os perfilhos da pastagem e, conseqüentemente, na eficácia dos inseticidas em atuar sobre as ninfas das cigarrinhas localizadas nas espumas. Nesse sentido, para melhor observação do desempenho das pontas de pulverização, os dados de deposição da calda pulverizada por unidade de área das lâminas de vidro foram matematicamente transformados, equiparando-se todos os tratamentos estudados em volumes de aplicação de 200 e $280 \mathrm{~L} \mathrm{ha}^{-1}$ (Figura 2), em que, por meio da razão entre as deposições médias, constituiu-se uma nota de índice de desempenho. Como cada $1 \mu \mathrm{L} \mathrm{cm}^{-2}$ de depósito monitorado nas lâminas corresponde a $100 \mathrm{~L} \mathrm{ha}^{-1}$ de calda, na Figura 2, as médias das deposições equiparadas em 200 e $280 \mathrm{~L} \mathrm{ha}^{-1}$ foram comparadas e constituiu-se o índice de desempenho para a relação entre os maiores e os menores volumes de 
aplicação. De acordo com o índice calculado, as pontas de pulverização posicionaram-se na seguinte ordem decrescente: TT11002-VS > AI11002-VS > TF-VP2 > TJ60-11002VS $\geq$ XR11002-VS > TX-VK4.

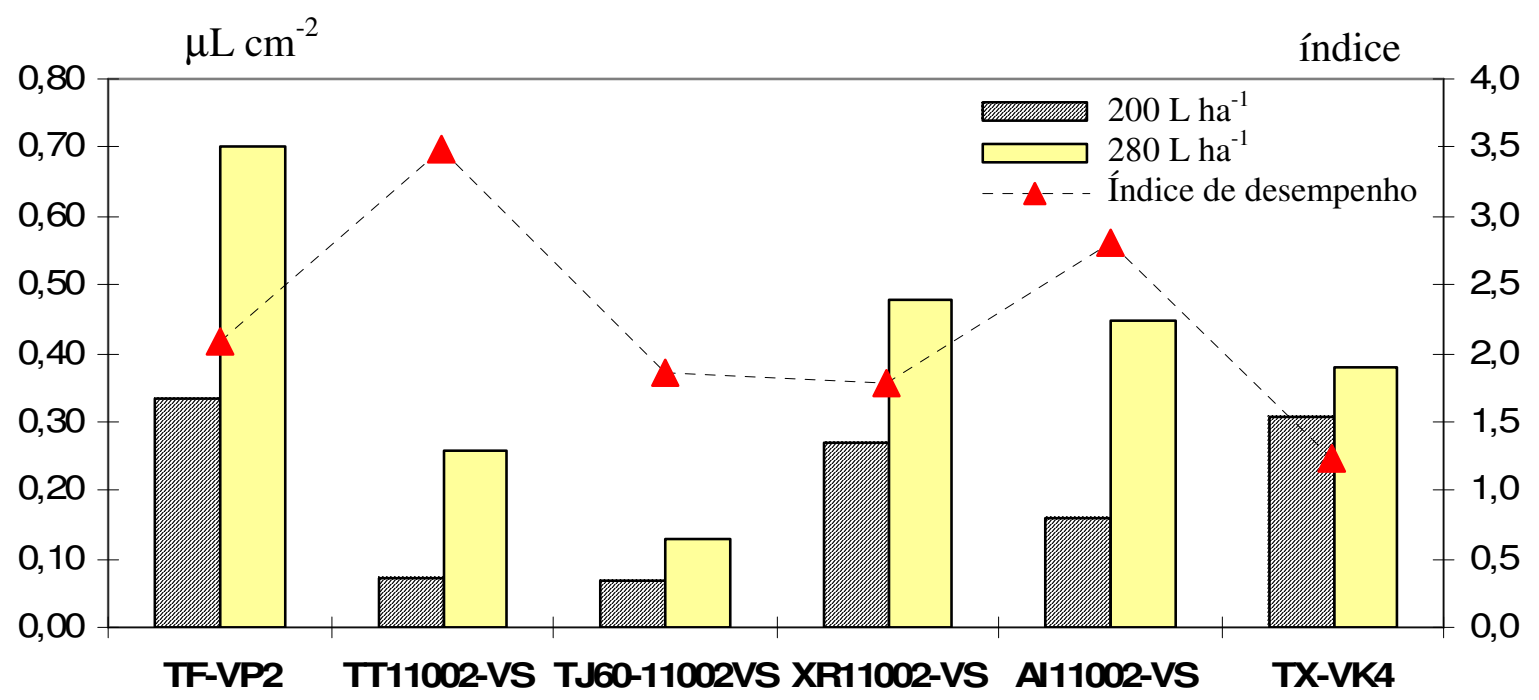

FIGURA 2. Deposição $\left(\mu \mathrm{L} \mathrm{cm}^{-2}\right)$ média da calda de pulverização nas lâminas de vidro posicionadas na base da pastagem de B. brizantha cv. MG-4 e índice de desempenho, em função das pontas e dos volumes de calda convertidos a 200 e $280 \mathrm{~L} \mathrm{ha}^{-1}$.

$\mathrm{Na}$ análise geral, os resultados indicam que o aumento da pressão de pulverização de 196 e $392 \mathrm{kPa}$, para as diferentes pontas estudadas, favoreceu o incremento do depósito da calda de pulverização nas porções superior e inferior dos perfilhos da $B$. brizantha cv. MG-4 e, principalmente, na região das espumas das cigarrinhas das pastagens. Entretanto, apesar do aumento da dose aplicada sobre o alvo desejado, pode-se, em alguns casos, como, por exemplo, para as pontas TX-VK4, XR110.02-VS e TJ110.02-VS, elevar em várias vezes as perdas por processos de deriva, devido à redução do tamanho das gotas que se formarão.

\section{CONCLUSÕES}

As pontas TF-VP2, XR11002-VS e AI11002-VS, nas pressões de 196 e $392 \mathrm{kPa}$, proporcionam as maiores deposições da calda de pulverização, na região das espumas das cigarrinhas das pastagens, apesar de apresentarem menor uniformidade na distribuição dos depósitos, nos referidos alvos, em relação aos tipos TX-VK4, XR110.02-VS e TJ110.02-VS.

O aumento da pressão de 196 para $392 \mathrm{kPa}$ promoveu aumento da deposição da calda de pulverização sobre a Brachiaria brizantha e na região onde se encontram as espumas das cigarrinhas das pastagens para todos os tipos de pontas de pulverização estudadas.

\section{REFERÊNCIAS}

ALMEIDA, R.G.; NASCIMENTO JUNIOR, D. Degradação, recuperação e sustentabilidade de pastagens cultivadas. Disponível em: <http://www.tdnet.com.br/domicio/Degradação_Giolo.htm.> Acesso em: 10 maio 2004.

ALVES, S.B. Controle biológico de pragas de pastagens. In: SIMPÓSIO SOBRE MANEJO DA PASTAGEM, 7., 1984, Piracicaba. Anais... Piracicaba: FEALQ, 1984. p.169-208.

DIAS FILHO, M.B. Pastagens cultivadas na Amazônia oriental brasileira: processos e causas de degradação e estratégias de recuperação. In: DIAS, L.E.; MELLO, J.W.V. Recuperação de áreas degradadas. Viçosa: DSO-UFV/SOBRADE, 1998. p.135-47. 
GALLO, D.; NAKANO, O.; SILVEIRA NETO, S.; CARVALHO, R.P.L.; BATISTA, G.C.; BERTI FILHO, E.; PARRA, J.R.P.; ZUCCHI, R.A.; ALVES, S.B.; VENDRAMIN, J.D.; MARCHINI, L.C.; LOPES, J.R.S.; OMOTO, C. Entomologia agrícola. Piracicaba: FEALQ, 2002. $920 \mathrm{p}$.

HISLOP, E.C.; COOKE, B.K.; HERRINGTON, P.M.; WESTERN, N.M.; WOODLEY, S.E. Efficient use of agrochemicals. Bristol: Long Ashton Research Station, 1987. p.48-9. Annual Report.

MELO, I.S.; AZEVEDO, J.L. Controle biológico. Jaguariúna: Embrapa, 1998. v.1, p.262.

NABINGER, C.; MARASCHIN, G.E.; MORAES, A. Pasture related problems in beef cattle production in southern Brazil. In: SIMPÓSIO INTERNACIONAL GRASSLAND

ECOPHYSIOLOGY AND GRAZING ECOLOGY, 1999, Curitiba. Anais... Curitiba: UFPR, 1999. p.23-48.

PALADINI, L.A. Metodologia para avaliação da deposição em pulverizações. 2000. $111 \mathrm{f}$. Tese (Doutorado em Proteção de Plantas) - Faculdade de Ciências Agronômicas, Universidade Estadual Paulista, Botucatu, 2000.

PEREIRA, J.R. Pragas e doenças em pastagens e forrageiras: curso de pecuária leiteira. Coronel Pacheco: EMBRAPA-CNPGL, 1990. 38 p. (Documentos, 45).

RAMOS, H.H.; PIO, L.C. Tecnologia de aplicação de produtos fitossanitários. In: ZAMBOLIM, L.; CONCEIÇÃ̃, M.Z.; SANTIAGO, T.. O que os engenheiros agrônomos devem saber para orientar o uso de produtos fitossanitários. Viçosa: UFV, 2003. p.133-201.

SERRÃO, A.S.; FALESI, I.C.; VEIGA, J.B. da; TEIXEIRA NETO, J.F. Produtividade de pastagens cultivadas em solos de baixa fertilidade das áreas de floresta do trópico úmido brasileiro. Belém: EMBRAPA-CPATU, 1978. 73 p.

SERRÃO, E.A.S.; UHL, C.; NEPSTAD, D.C. Deforestation for pasture in the humid tropics: is it economically and enviromentally sound in the long term? In: INTERNATIONAL GRASSLAND CONGRESS, 17., 1993, Rockhampton. Proceedings... Palmerston North: New Zealand Grassland Association, 1993. p.2215-21.

SILVA, M.A.S. Depósitos da calda de pulverização no solo e em plantas de tiririca (Cyperus rotundus L.) em diferentes condições de aplicação. 2000. $57 \mathrm{f}$. Tese (Doutorado em Agricultura) Faculdade de Ciências Agronômicas, Universidade Estadual Paulista, Botucatu, 2000.

SILVEIRA NETO, S. Controle de insetos nocivos às pastagens de Brachiaria spp. In: SIMPÓSIO SOBRE MANEJO DA PASTAGEM, 11., 1994, Piracicaba. Anais... Piracicaba: FEALQ, 1994. p.73-97.

SOUZA, R.T. Efeito da eletrização de gotas sobre a variabilidade dos depósitos de pulverização e eficácia do glyphosate no controle de plantas daninhas na cultura da soja. 2002. 69f. Tese (Doutorado em Agricultura) - Faculdade de Ciências Agronômicas, Universidade Estadual Paulista, Botucatu, 2002.

SPRAYING SYSTEMS Co. Teejet Spray Products. Guia do Comprador 201-BR/P. 2000. 29 p.

SPRAYING SYSTEMS Co. Produtos de Pulverização para Agricultura. Catálago 46M-BR/P. 1998. $104 \mathrm{p}$.

TOMAZELA, M.S. Efeitos do estádio de desenvolvimento de Brachiaria plantaginea (Link) Witch, ângulo de aplicação e tipo de ponta na deposição da calda de pulverização. 2000. $53 \mathrm{f}$. Tese (Doutorado em Agricultura) - Faculdade de Ciências Agronômicas, Universidade Estadual Paulista, Botucatu, 2000.

VALÉRIO, J.R.; KOLLER, W.W. Proposição para o manejo integrado das cigarrinhas-daspastagens. Campo Grande: EMBRAPA-CNPGC, 1995. 37 p. (Documentos, 52). 
VELINI, E.D. Estudos e desenvolvimento de métodos experimentais e amostrais adaptados à matologia. 1995. 250 f. Tese (Doutorado em Agricultura) - Faculdade de Ciências Agrárias e Veterinárias, Universidade Estadual Paulista, Jaboticabal, 1995.

ZIMMER, A.H.; CORRÊA, E.S.A pecuária nacional: uma pecuária a pasto? In: ENCONTRO SOBRE RECUPERAÇÃO DE PASTAGENS, 1993, Nova Odessa. Anais... Nova Odessa: Instituto de Zootecnia, 1993. p.1-25.

ZIMMER, A.H.; EUCLIDES FILHO, K. As pastagens e a pecuária de corte brasileira. In: SIMPÓSIO INTERNACIONAL SOBRE PRODUÇÃO ANIMAL EM PASTEJO, 1997, Viçosa. Anais... Viçosa: UFV, 1997. p.349-79. 